\title{
Scaling of Glycine Nucleation Kinetics with Shear Rate and Glass- Liquid Interfacial Area
}

\author{
Carol Forsyth, Iain S. Burns, Paul A. Mulheran, and Jan Sefcik* \\ Department of Chemical and Process Engineering, University of Strathclyde, 75 Montrose Street, Glasgow, G1 1XJ, United Kingdom \\ Supporting Information
}

ABSTRACT: The scaling of the nucleation kinetics of glycine was investigated in supersaturated aqueous solutions under isothermal conditions. Induction times were measured in a Couette cell with a wide range of average shear rates $\dot{\gamma}_{\text {avg }}\left(25-250 \mathrm{~s}^{-1}\right)$ and a range of glass-liquid interfacial areas A $\left(2.5-10 \mathrm{~cm}^{2}\right.$ per $\mathrm{mL}$ solution). The probability distributions of induction times were found to scale with shear rate and glass-liquid interfacial area, with the characteristic time scale $\left(\dot{\gamma}_{\text {avg }} A\right)^{-1}$. Primary nucleation rates and growth times to reach detection (estimated from the probability distributions) were both dependent on this time scale. In-situ dynamic light scattering revealed mesoscale clusters in the solutions that increased in size over time at rates which also depended on this time scale. The increase in size was thought to be due to the shear-enhanced aggregation or coalescence of mesoscale clusters leading to a higher number of larger mesoscale clusters,

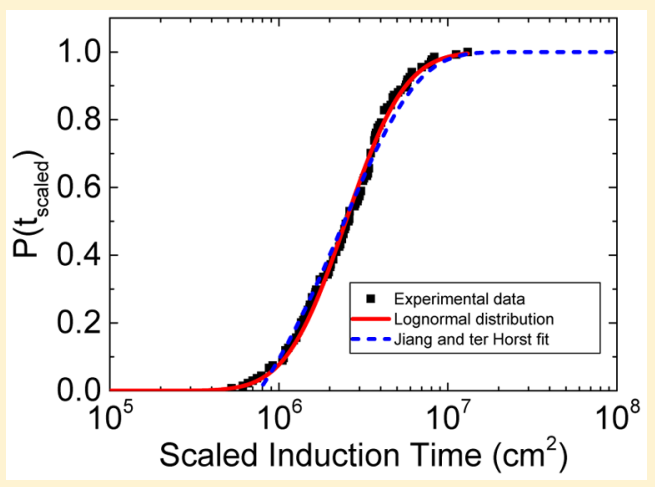
resulting in higher rates of primary nucleation.

\section{INTRODUCTION}

Fluid shear has been reported to influence the nucleation of crystals from solution. It is well-known to have an effect on secondary nucleation since shear forces imposed on a crystal face can be sufficient to produce secondary nuclei from the crystal surface, ${ }^{1,2}$ but the effect that fluid shear can have on primary nucleation from solution is not well understood to date, particularly for small organic molecules. ${ }^{3,4}$ Studies have reported that fluid shear can influence the primary nucleation of various organic compounds from solution, including protein molecules such as lysozyme ${ }^{5-7}$ and insulin $^{8}$ and smaller molecules such as glycine ${ }^{4,9}$ carbamazepine, ${ }^{10}$ butyl paraben, ${ }^{3}$ and $m$-hydroxybenzoic acid. ${ }^{11}$ In some cases, fluid shear can enhance primary nucleation rates, ${ }^{3,4,9-11}$ while in others, it can suppress them, ${ }^{3,5-7}$ and the mechanisms behind the role of fluid shear on primary nucleation therefore remain unclear.

In many studies on the influence of fluid shear on nucleation, the shear rates were not well quantified so in our previous work, ${ }^{4}$ Couette and capillary flow devices were used to achieve controlled, quantifiable flow conditions. It was shown that this controlled fluid shear could be used to significantly enhance primary nucleation rates in supersaturated glycine solutions. ${ }^{4}$ Controlled fluid shear in a Couette cell has been found to enhance primary nucleation in other systems, including butyl paraben $^{3}$ and $m$-hydroxybenzoic acid. ${ }^{11}$ While it is recognized that complex flow fields are present in industrial crystallization processes, it is useful to understand the effect of simple, wellcontrolled flow fields on various aspects of the crystallization process, such as primary nucleation, and then map this information on more complex equipment through knowledge of shear rate distributions obtained for example by computational fluid dynamics.

In addition to fluid shear, exposure of solutions to a solid surface such as the walls of the glass Couette cell is known to have an influence on nucleation. ${ }^{1,12}$ It is widely claimed that true homogeneous nucleation is uncommon, so nucleation that occurs heterogeneously is most likely. ${ }^{1}$ The overall free energy barrier for heterogeneous nucleation is lower than the corresponding free energy barrier for homogeneous nucleation, and the overall free energy barrier for heterogeneous nucleation decreases with increasing surface wettability. ${ }^{13}$ The effect of interfacial area had not been explored in our previous research. In the present work, supersaturated glycine solutions were also exposed to a range of different glass-liquid interfacial areas to investigate the effect on nucleation kinetics. The results of repeated experiments allowed a statistical analysis to be performed, and the possibility of the measured induction times scaling with shear rate and surface area was investigated.

The second element of this paper involves gaining a better understanding of the mechanism by which nucleation takes place. Classical nucleation theory has some well-known shortcomings, and experimental and theoretical work has led to the proposal of alternative theories, including a two-step nucleation process. ${ }^{14-18}$ The two-step mechanism involves the production of intermediate, disordered, metastable liquid-like clusters of solute molecules, in which solute molecules then order themselves into crystalline nuclei. ${ }^{14-18}$

Received: July 22, 2015

Revised: November 5, 2015 


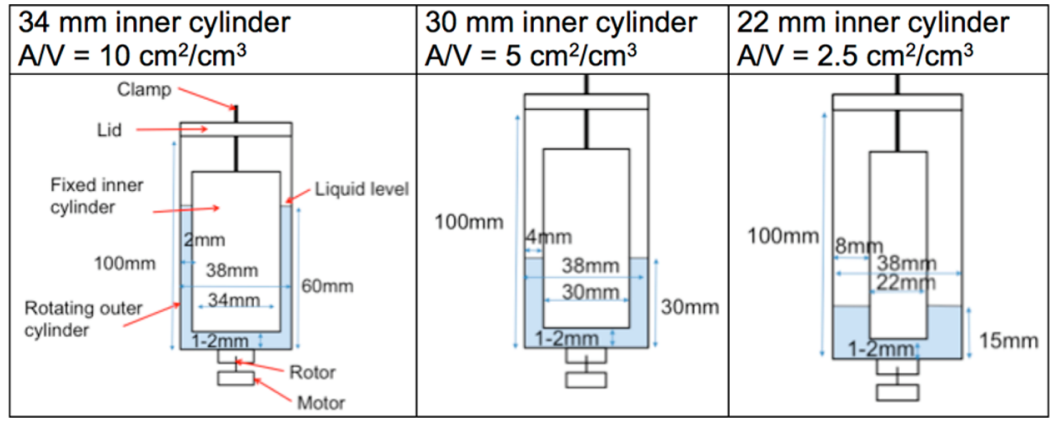

Figure 1. Diagrams of the three Couette setups used to achieve different surface areas for a fixed volume of solution ( $15 \mathrm{~mL}$ in all cases).

A previous study on glycine nucleation reported that glycinerich mesoscale clusters (nanodroplets) of around $250 \mathrm{~nm}$ diameter with liquid-like properties exist in aqueous glycine solutions. ${ }^{9,19}$ These were not thought to be directly involved in productive crystal nucleation but could coalesce to larger clusters to give access to a more rapid nucleation pathway. ${ }^{9}$ It was proposed that productive nucleation of glycine was most likely to occur in large glycine-rich clusters with a diameter greater than about $750 \mathrm{~nm}$, which were only observed in stirred solutions.

The aggregation of clusters has been proposed to play a role in other recent studies on nucleation. For example, supersaturated solutions of calcium carbonate were found to contain distinct cluster sizes, and this was interpreted as nucleation taking place by cluster aggregation. ${ }^{15,20}$ The study of butyl paraben solutions undergoing flow in a Couette cell ${ }^{3}$ concluded that enhanced cluster aggregation was a plausible explanation for the enhanced nucleation rates seen under flow, and a further study on the influence of Couette flow on m-hydroxybenzoic $\operatorname{acid}^{11}$ nucleation was also supportive of the theory of enhanced cluster aggregation. A study on the effect of mixing on mesoscale clusters in DL-valine solutions suggested that mixing resulted in the presence of larger mesoscale clusters than in quiescent solutions, and the presence of larger clusters corresponded to faster nucleation. The larger clusters were thought to form through shear-induced coalescence or surfaceassisted coalescence. ${ }^{21}$

In this paper, in situ dynamic light scattering (DLS) measurements of glycine solutions that had been sheared were taken to obtain information on the size of mesoscale clusters in the solutions and how the sizes were affected by fluid shear and glass-liquid interfacial area. These findings were then compared with the induction time results to investigate the relationship between mesoscale cluster size and induction time, leading to a better understanding of the prevailing nucleation mechanism.

\section{EXPERIMENTAL SECTION}

All experiments were carried out using supersaturated aqueous glycine solutions in a Couette flow cell. Two main measurements were carried out: (1) induction time measurements and (2) in situ DLS measurements.

Solution Preparation. Glycine (CAS number 56-40-6) of $\geq 99 \%$ purity (Sigma-Aldrich item G8898) was used without further purification. Solutions were prepared by dissolving glycine in deionized water. Two concentrations, $c$, of solution were prepared - $307 \mathrm{~g}$ glycine $/ \mathrm{kg}$ water and $325 \mathrm{~g}$ glycine $/ \mathrm{kg}$ water. Solutions were prepared in sealed glass bottles and were stirred at $55^{\circ} \mathrm{C}$ for $24 \mathrm{~h}$ to ensure that glycine was fully dissolved. For these concentrations of glycine in water at $55{ }^{\circ} \mathrm{C}$, solutions were undersaturated. During experiments, the 307 $\mathrm{g} / \mathrm{kg}$ and $325 \mathrm{~g} / \mathrm{kg}$ solutions were cooled to 20 and $23^{\circ} \mathrm{C}$ respectively. These temperatures and concentrations were chosen so that the level of supersaturation was the same for the two solutions (the small temperature difference was assumed to have a negligible influence on nucleation rates). The solubility, $c^{*}$, of glycine is $219 \mathrm{~g}$ glycine $/ \mathrm{kg}$ water at $20{ }^{\circ} \mathrm{C}$ and $232 \mathrm{~g}$ glycine $/ \mathrm{kg}$ water at $23{ }^{\circ} \mathrm{C}$. ${ }^{36}$ Cooling therefore resulted in a supersaturation, $S$, of 1.40 for both solutions, where

$$
S=\frac{c}{c^{*}}
$$

Because of the $\mathrm{pH}$ of the glycine solutions used, the $\alpha$-glycine polymorph was expected to form during the experiments, ${ }^{22}$ and this was confirmed by XRD.

Couette Flow Setup. Nucleation experiments were performed using a cylindrical Couette flow geometry. To allow the role of the glass surface to be studied, the area of the Couette cell surface in contact with the liquid was varied for a fixed volume of supersaturated glycine solution. This was achieved by changing the diameter of the Couette cell's inner cylinder as shown in Figure 1. Supersaturated glycine solutions were subjected to controlled flow conditions in the Couette flow setups. The Couette flow setup was similar to what was used in our previous work, ${ }^{4}$ but in addition to using a $30 \mathrm{~mm}$ diameter inner cylinder, $22 \mathrm{~mm}$ and $34 \mathrm{~mm}$ diameter inner cylinders were also used. More details on the Couette flow setup geometries are given in the Supporting Information.

The inner cylinders were kept fixed, while the outer cylinder was rotated using a motor powered by a variable DC power supply. The fixed inner/rotating outer cylinder geometry does not result in the Taylor instabilities (Taylor vortices superimposed on laminar Couette flow ${ }^{4,23}$ ), which would have occurred in a fixed outer/rotating inner cylinder for the conditions used. ${ }^{4,23}$ This meant that the laminar shear rates in the gap between the two cylinders could be quantified by solving the Navier-Stokes equation.

The velocity profile across the vertical gap of the Couette cell was approximated by solving a momentum balance across the system (from the Navier-Stokes equation) assuming steady state operation. The shear rate, $\dot{\gamma}$, profile across the gap could be found from the derivative of the velocity profile with respect to radial position, $r$. The average shear rate across the gap, $\dot{\gamma}_{\text {avg }}$ was calculated from

$$
\dot{\gamma}_{\text {avg }}=\frac{1}{r_{\text {out }}-r_{\text {in }}} \int_{r_{\text {in }}}^{r_{\text {out }}} \dot{\gamma}(r) \mathrm{d} r=\frac{2 \pi N r_{\text {out }}}{r_{\text {out }}-r_{\text {in }}}
$$

Here $N$ is the rotational rate, $r_{\text {out }}$ is the radius of the outer cylinder, and $r_{\text {in }}$ is the radius of the inner cylinder. The maximum and minimum shear rates found to occur for a particular set of conditions varied by a maximum of $15 \%$ from the average shear rate. The maximum shear rate occurred at the inner cylinder walls. The range of average shear rates that was investigated for each setup is given in Table 1 . The maximum shear rate was limited by the need to maintain mechanical stability to ensure that the cylinders were accurately concentrically aligned.

Using the velocity of the rotating outer cylinder and the distance between the vertical cylinder walls, the range of Reynolds number (eq 
Table 1. Range of Average Shear Rates for Each Setup and the Resulting Range of Reynolds Numbers

\begin{tabular}{cccc}
$\begin{array}{c}\text { gap } \\
(\mathrm{mm})\end{array}$ & $\begin{array}{c}\text { surface area per mL solution } \\
\text { contained within vertical cylinder } \\
\text { gap }\left(\mathrm{cm}^{2} / \mathrm{cm}^{3}\right)\end{array}$ & $\begin{array}{c}\text { average shear } \\
\text { rate range } \\
\left(\mathrm{s}^{-1}\right)\end{array}$ & $\begin{array}{c}\text { Reynolds } \\
\text { number range }\end{array}$ \\
8 & 2.5 & $25-125$ & $1100-5500$ \\
4 & 5 & $25-250$ & $280-2800$ \\
2 & 10 & $25-250$ & $70-700$ \\
\hline
\end{tabular}

3), $R e$, investigated with each setup is given in Table 1 . The kinematic viscosity, $\eta$, was obtained by experiment and compared well to correlations found in the literature. ${ }^{24}$

$$
R e=\frac{\left(2 \pi N r_{\text {out }}\right)\left(r_{\text {out }}-r_{\text {in }}\right)}{\eta}
$$

Critical Reynolds numbers for transition from laminar to complex flow depend on the geometry used and can be found experimentally. To assess whether the flow appeared to be steady for the experimental conditions used, rheoscopic fluid (suspension of mica platelets that align with the flow) and dye injections were used in the Couette cell, and the flow was visualized for the range of shear rates used in each experimental setup. The flow appeared to remain steady visually, which is consistent with previously published studies, where the laminar Couette regime is only observed when the inner cylinder is stationary. ${ }^{25}$ We note that large instabilities were observed when the inner cylinder was rotated and the outer cylinder was kept fixed, in agreement with what is expected from theory ${ }^{23}$ and with previous experimental observations, ${ }^{25}$ but these conditions were not used for experiments reported here.

Induction Time Measurement Setup. Couette flow experiments were monitored by one of two methods: (1) measurements of transmitted light intensity; (2) imaging of the nucleation process followed by image analysis. The layout is given in Figure 2.

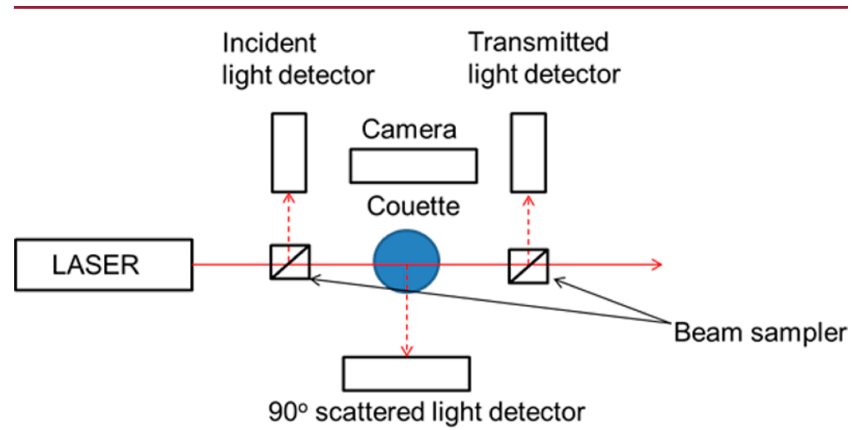

Figure 2. Diagram of monitoring equipment used to measure induction times.

Transmission data were obtained using a custom-built instrument, similar to one used previously for in situ monitoring of stirring effects on the crystallization of carbamazepine ${ }^{10}$ and for measuring the induction times of glycine solutions undergoing shear in a Couette cell. ${ }^{4}$ When the induction time was reached, solutions became turbid over a relatively short time scale (as short as around $10 \mathrm{~s}$ ), leading to a sharp decrease in transmission and an increase in mean pixel intensity. Induction times were obtained by finding the point of inflection of the rising (or falling) signals. The measurement methods were found to give consistent results (for imaging, the mean induction times were similar to those obtained through transmission measurements for identical experimental conditions ${ }^{4}$ ). We note that the majority of experiments carried out were monitored through transmission measurements; imaging was only done for a small number of experiments using the $30 \mathrm{~mm}$ inner cylinder setup. ${ }^{4}$

More information on the transmission and imaging setups is given in the Supporting Information.
Induction Time Measurement Procedure. Induction time measurements were carried out for a wide range of shear rates and surface areas, as shown in Figure 3. Since each shear rate (with the

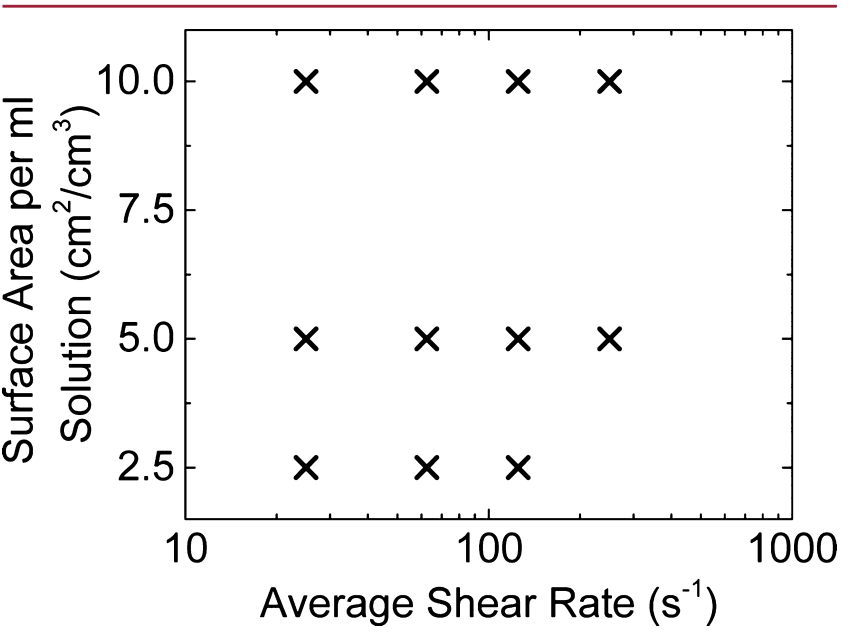

Figure 3. Diagram showing the different combinations of shear rate and glass-liquid interfacial area per unit solution volume used in the induction time experiments.

exception of $250 \mathrm{~s}^{-1}$ ) was repeated for each cylinder geometry, a robust Design of Experiments analysis could be carried out on the data. Experiments were repeated at least 10 times. Ten repetitions have proven to be sufficient to allow reliable statistical trends to be obtained from induction time data that was measured through a similar method before. $^{4}$ Details of the experimental procedure are given in the Supporting Information.

Dynamic Light Scattering Setup. To allow in situ DLS measurements to be made, hardware was set up as shown in Figure 4. The beam emerging from the laser was focused from $1 \mathrm{~mm}$ diameter

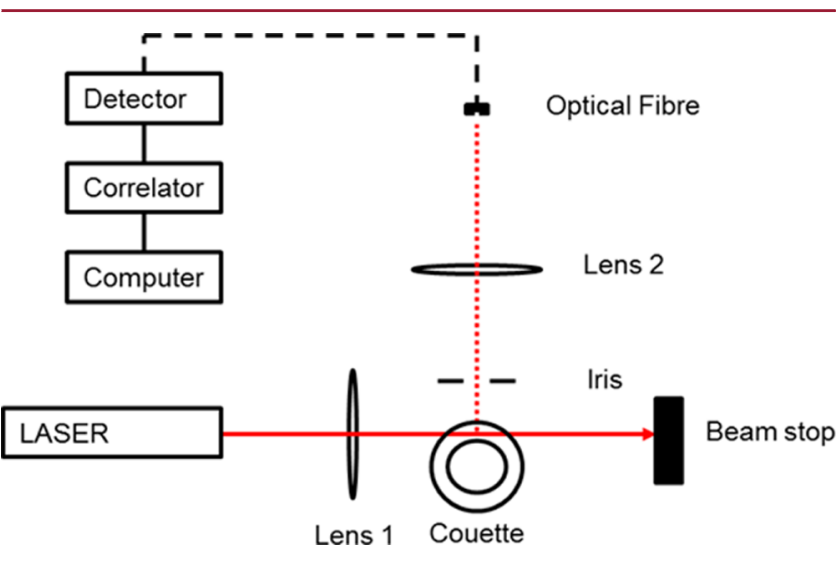

Figure 4. Diagram of monitoring equipment used for DLS measurements.

to a narrow beam waist of $\sim 100 \mu \mathrm{m}$ diameter. This was necessary to obtain a small enough measurement volume (calculated from cross section of beam and external aperture of the optical fiber) so that local fluctuations in scattering intensity would not be lost through averaging. The beam was focused at the center of the gap between the vertical inner and outer cylinder walls. Light scattered at $90^{\circ}$ was imaged onto the plastic external aperture of optical fiber and collected by optical fiber. The light was imaged onto the fiber at 1:1 magnification. A $2 \mathrm{~mm}$ iris was present close to the Couette cell to stop strong reflections from reaching the fiber and limit the range of angles reaching the detector.

The optical fiber guided the scattered light to a detector (Excelitas Single Photon Counting Module, SPCM-AQRH-14-FC). The 
resulting signal was processed by a digital correlator (ALV-7004/ USB), and the data were recorded using a standard PC.

Dynamic Light Scattering Couette Procedure. DLS measurements at $90^{\circ}$ were carried for a range of shear rate and surface area, as shown in Figure 5. The DLS procedure involved shearing a solution

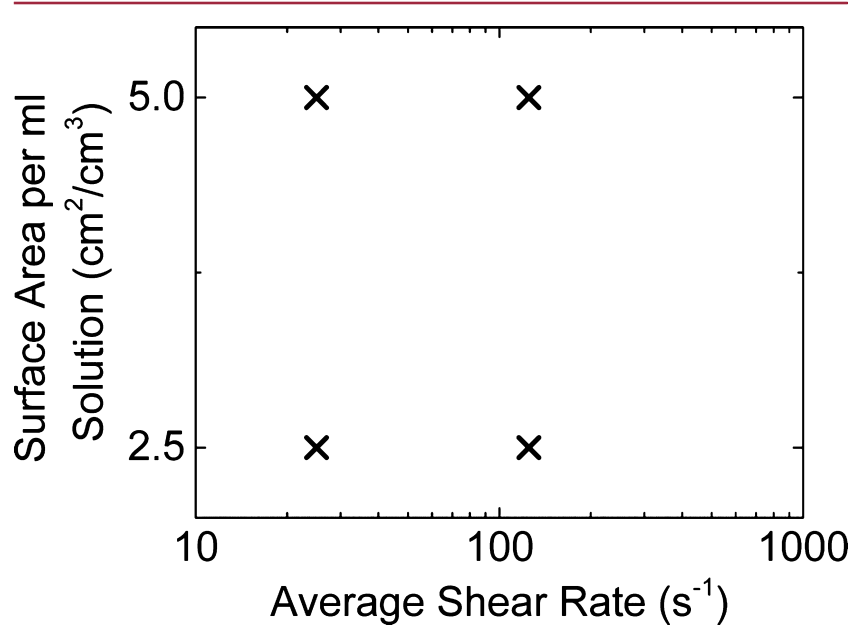

Figure 5. Diagram showing the different combinations of shear rate and glass-liquid interfacial area per unit solution volume used in the DLS experiments.

for a chosen duration of time, stopping shearing (gradually slowing down the motor to zero $\mathrm{rpm}$ ) and then taking DLS measurements. The resulting intensity autocorrelation functions $\left(g_{2}(\tau)-1\right)$ were analyzed through a cumulant analysis ${ }^{26}$ to obtain the average hydrodynamic radius of clusters in the solutions. The experimental procedure was similar to the one used for induction time measurements, but rather than shearing the solution until it became clouded by crystals, here the solution was sheared for shorter durations. More information is given in the Supporting Information.

\section{RESULTS AND DISCUSSION}

Induction Time Measurements. Induction time measurements are shown in Figure 6. Increasing the shear rate led to shorter average induction times for every cylinder surface arrangement studied. For each shear rate studied, cylinder arrangements which exposed the glycine solution to larger surface areas resulted in shorter average induction times than cylinder arrangements with smaller surface areas. A Design of Experiments statistical analysis was carried out, and this is detailed in the Supporting Information.

The induction times measured for sheared solutions were considerably less than those measured for unsheared solutions for each shear rate used with the $10 \mathrm{~cm}^{2} / \mathrm{cm}^{3}$ cylinder arrangement; however, the induction times measured for $25 \mathrm{~s}^{-1}$ experiments using the $2.5 \mathrm{~cm}^{2} / \mathrm{cm}^{3}$ and $5 \mathrm{~cm}^{2} / \mathrm{cm}^{3}$ cylinder arrangements were fairly close to the unsheared induction times.

It is noted that induction times for the unsheared solutions were detected by visually observing solutions with the naked eye, while induction times for sheared solutions were obtained through the quantitative detection method described. The rate at which turbidity increased with the $25 \mathrm{~s}^{-1}$ experiments using the $2.5 \mathrm{~cm}^{2} / \mathrm{cm}^{3}$ and $5 \mathrm{~cm}^{2} / \mathrm{cm}^{3}$ cylinder arrangements was lower than for all of the other experimental setups, suggesting slower secondary nucleation. Sufficient secondary nucleation had to take place before measured signals were affected, meaning that an overestimation of induction times may have

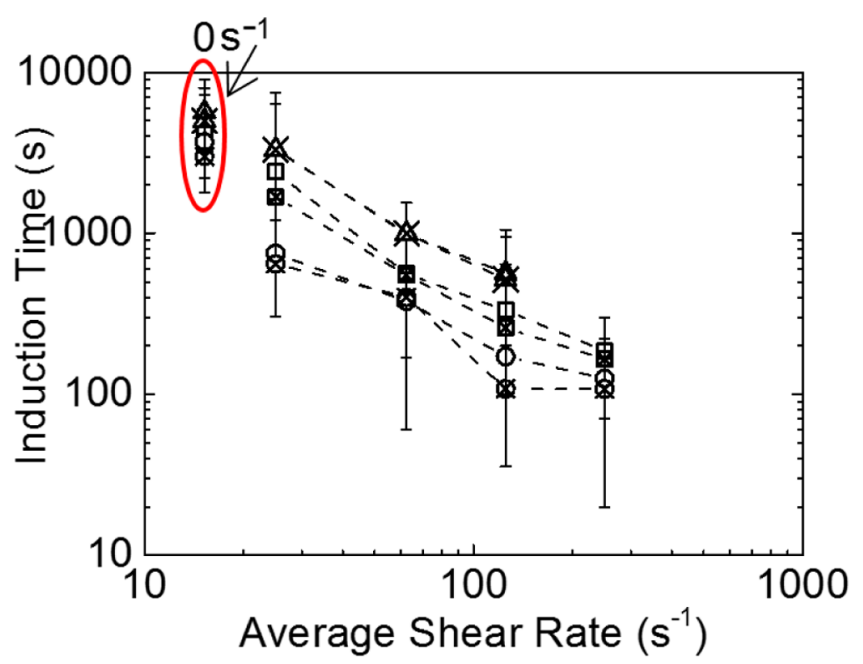

Figure 6. Mean (open symbols) and median (crossed symbols) induction times measured for each shear rate/cylinder arrangement. Triangles, squares and circles represent surface to volume ratios of 2.5 $\mathrm{cm}^{2} / \mathrm{cm}^{3}, 5 \mathrm{~cm}^{2} / \mathrm{cm}^{3}$ and $10 \mathrm{~cm}^{2} / \mathrm{cm}^{3}$, respectively. Error bars represent the full spread of measured induction times. Note that circled data-points were recorded without shear and are therefore not defined on the $x$-axis.

occurred for the $25 \mathrm{~s}^{-1}$ experiments using the $2.5 \mathrm{~cm}^{2} / \mathrm{cm}^{3}$ and $5 \mathrm{~cm}^{2} / \mathrm{cm}^{3}$ cylinder arrangements. ${ }^{4}$

Distributions of Induction Times. The distributions of induction times obtained were further investigated to gain a better understanding of underlying nucleation phenomena. In our previous study on nucleation of glycine undergoing shear in a Couette flow cell, it was found that a log-normal distribution of induction times or a distribution proposed by Jiang and ter Horst $^{27}$ fitted the experimental distribution of induction times well. ${ }^{4}$ We now verify that these distributions fit the experimental data obtained here (see Supporting Information).

The model proposed by Jiang and ter Horst ${ }^{27}$ allowed us to obtain quantitative insight into nucleation phenomena from experimentally observed distributions of induction time. With the model, the cumulative distribution function of induction time, $P\left(t_{i}\right)$, could be written as

$$
P\left(t_{\mathrm{i}}\right)=1-\exp \left(-J V\left(t_{\mathrm{i}}-t_{\mathrm{g}}\right)\right)
$$

Here $J$ is the nucleation rate per unit volume, $V$ is the volume of the solution (a volume of $15 \mathrm{~mL}$ was used in the experiments.), $t_{\mathrm{i}}$ is the induction time measured by experiment, and $t_{\mathrm{g}}$ is the growth time. The growth time is interpreted as the time taken after the formation of a nucleus for the crystal(s) to reach a detectable level, for example, through extensive secondary nucleation due to a primary crystal.

The best fit values for $J$ and $t_{\mathrm{g}}$ (eq 4) were found for each setup and are given in Figure 7 and Figure 8 respectively. It was already seen that the observed induction times decrease with increasing shear rate and surface area (cf. Figure 6). This is in agreement with what is shown in Figure 7 and Figure 8. It can be seen that nucleation rates can be enhanced by at least 1 order of magnitude by laminar shear. It can be also seen that growth times decrease significantly with increasing shear rates. Nucleation rates are shown to increase with increasing surface area, and growth times are shown to decrease with increasing surface area. 


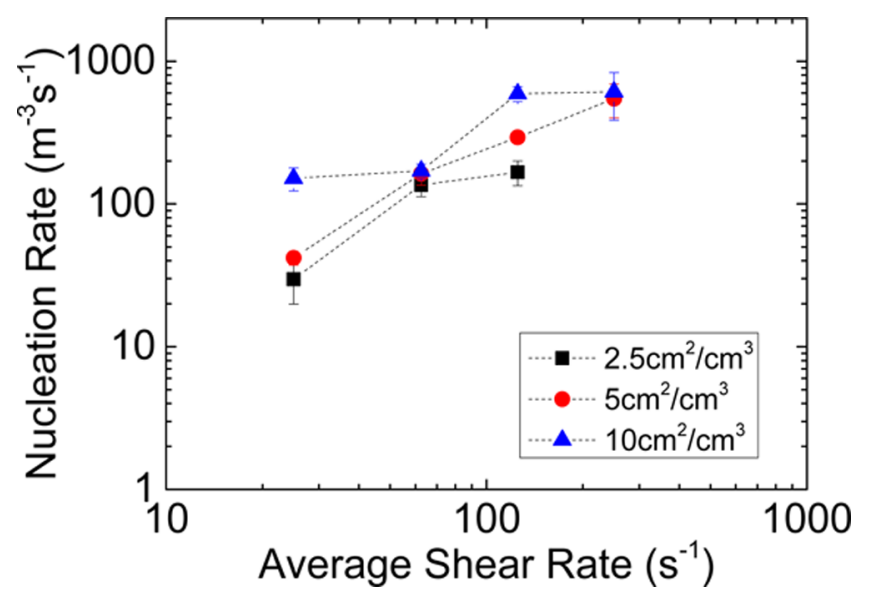

Figure 7. Nucleation rates $J$ estimated from the best fit of the model by Jiang and ter Horst. Error bars represent $95 \%$ confidence intervals.

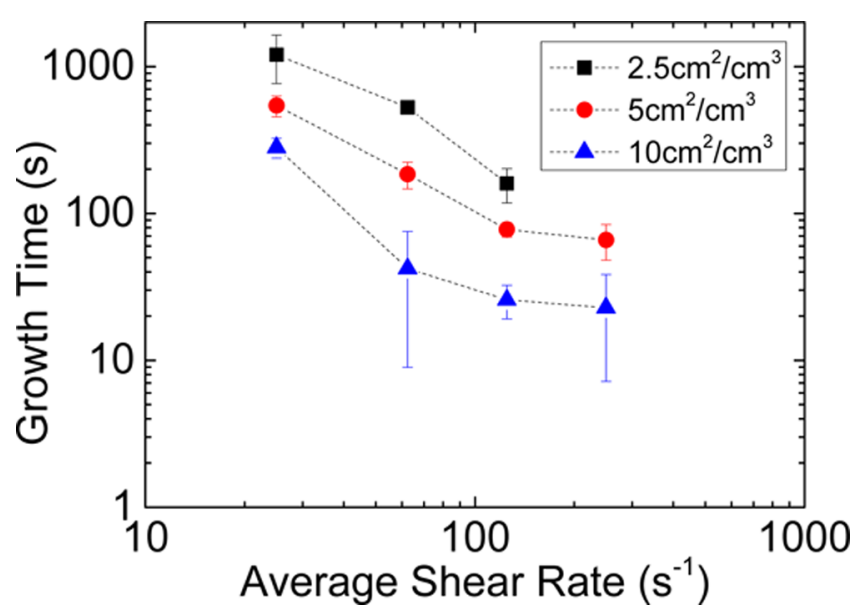

Figure 8. Growth times $t_{\mathrm{g}}$ estimated from the best fit of the model by Jiang and ter Horst. Error bars represent 95\% confidence intervals.

Scaling of Induction Times. Scaling was investigated to see whether the distributions of induction times appeared to scale by shear rate and surface area.

The induction times were scaled by both the average shear rate and the surface area, and the cumulative probability distributions of the scaled times were found.

$$
t_{\text {scaled } \mathrm{i}}=\dot{\gamma}_{\text {avg }} t_{\mathrm{i}} A=\gamma A
$$

Here $\gamma$ is the total strain.

All of the cumulative probability distributions for each set of conditions converged as shown in Figure 9. This suggested that induction times were governed by one key time scale, proportional to $\left(1 /\left(\dot{\gamma}_{\text {avg }} A\right)\right)$. When dealing with the stochastic nucleation of a crystal, the time scale appears rational as it would be expected that nucleation rates could be enhanced by increased shear rates ${ }^{3-5}$ and surface areas. ${ }^{12,13}$ The time scale, however, is more difficult to rationalize with the nonstochastic growth phase, particularly why a larger surface area would result in shorter growth times. This is discussed more in the following sections.

An overall cumulative distribution function (CDF) of the scaled data is shown in Figure 10. Since the log-normal and Jiang and ter Horst models fitted the distributions of induction times well, they were also used to produce master fits to the scaled induction time data, as shown in Figure 10. The scaled

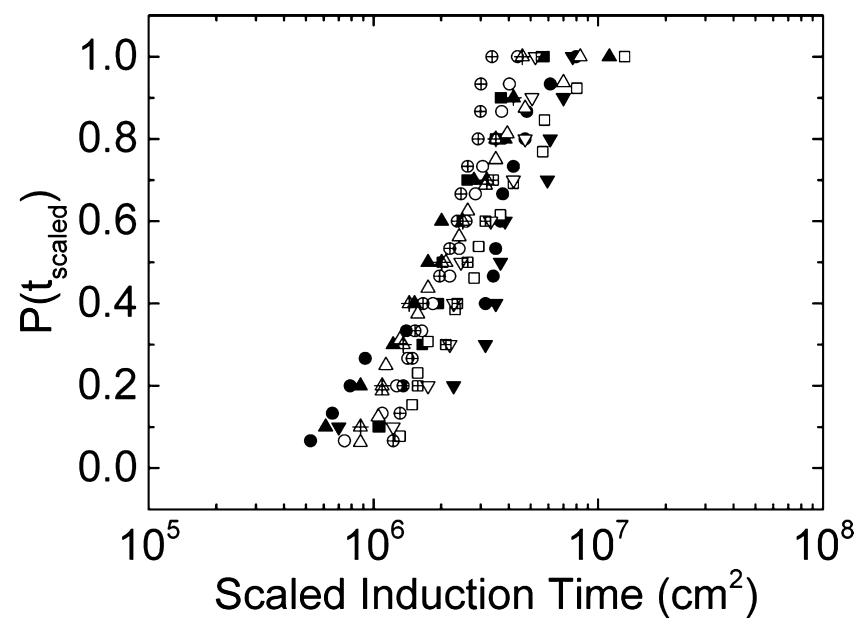

Figure 9. Experimental cumulative distribution functions of induction times for each setup scaled by the average shear rate and surface area. Squares, circles, pyramids and inverted pyramids represent shear rates of $25 \mathrm{~s}^{-1}, 62.5 \mathrm{~s}^{-1}, 125 \mathrm{~s}^{-1}$ and $250 \mathrm{~s}^{-1}$, respectively. Crossed, open and filled symbols represent surface to volume ratios of $2.5 \mathrm{~cm}^{2} / \mathrm{cm}^{3}, 5$ $\mathrm{cm}^{2} / \mathrm{cm}^{3}$ and $10 \mathrm{~cm}^{2} / \mathrm{cm}^{3}$, respectively.

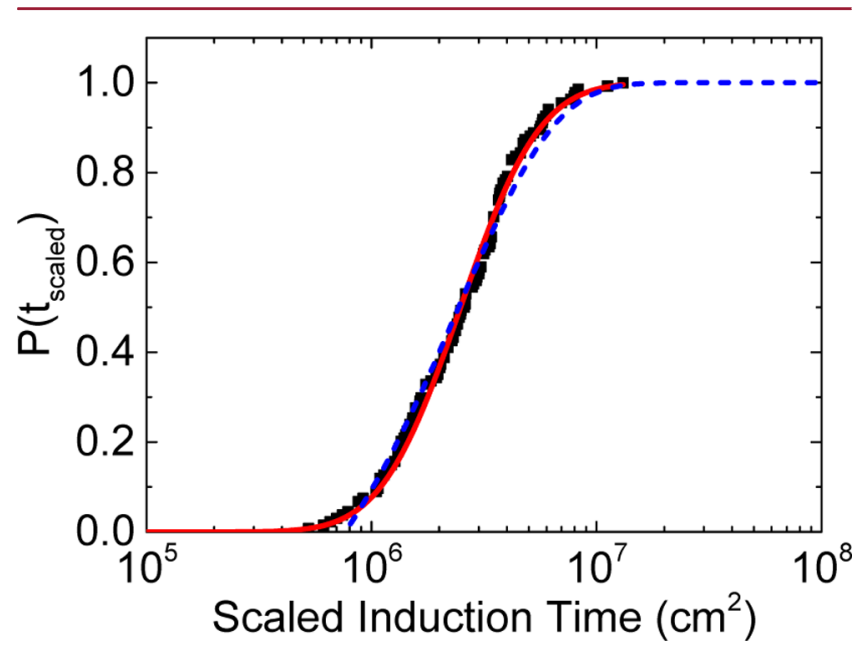

Figure 10. Overall experimental cumulative distribution function of scaled induction times (symbols) and cumulative distributions based on log-normal (solid line) and Jiang and ter Horst (dashed line) models fitted to the data.

equations provided a good fit to the individual experimental CDFs, with $R^{2}=0.88$ and $R^{2}=0.87$ respectively.

For the Jiang and ter Horst model, eq 4 was written in terms of the scaled induction times and fitting parameters $\alpha$ and $\beta$ :

$$
P\left(t_{\text {scaled i }}\right)=1-e^{-\alpha V\left(t_{\text {scaled i }}-\beta\right)}
$$

A scaled equation was obtained by finding the best fit values of $\alpha$ and $\beta$ (eq 6) to the overall CDF produced from all of the scaled data (Figure 10). Nucleation rates and growth times could be obtained from the fitting parameters $\alpha$ and $\beta$ using the scaling factor $\psi\left(\psi=\dot{\gamma}_{\text {avg }} A\right)$, e.g., $J=\alpha \psi$ and $t_{\mathrm{g}}=(\beta / \psi)$.

This analysis therefore suggested that when induction times were scaled by shear rate and surface area, probability distributions converged and scaled equations, based on lognormal distributions of induction times or the model given by Jiang and ter Horst, provided good fits to the data. It should be noted that the two methods (log-normal and Jiang and ter Horst model) make very different assumptions about the 
independence of nucleation events. The differences essentially stem from the independence of events (Poisson/Jiang and ter Horst $^{27,28}$ ) versus their dependence (resulting in a log-normal distribution $^{29}$ ).

Figure 11 shows the nucleation rates $(J)$ and growth times $\left(t_{\mathrm{g}}\right)$ obtained using the model proposed by Jiang and ter Horst $^{27}$ (see Figure 7 and Figure 8) plotted as a function of $\dot{\gamma}_{\text {avg }} A$.

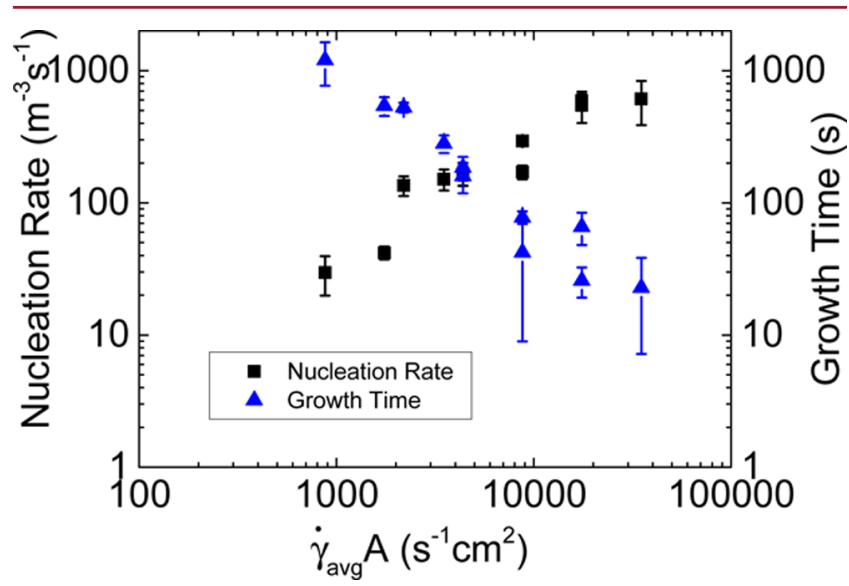

Figure 11. Nucleation rates $J$ and growth times $t_{\mathrm{g}}$ estimated from the best fit of the model by Jiang and ter Horst plotted as a function of the product of the average shear rate and surface area. Error bars represent $95 \%$ confidence intervals.

Dynamic Light Scattering. Examples of autocorrelation functions that were obtained from DLS measurements are given in Figure 12. The graph shows the average of the 25

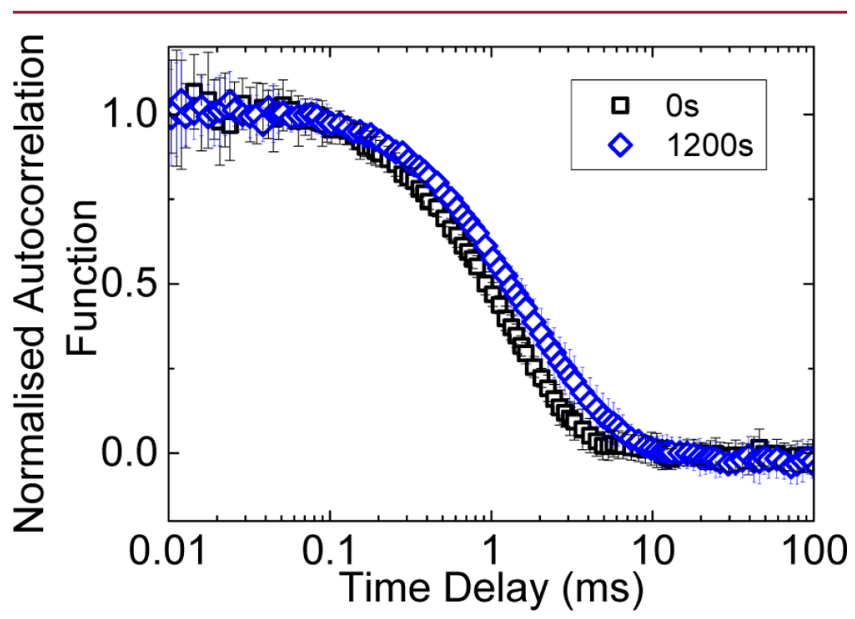

Figure 12. Average of the 25 autocorrelation functions measured after 0 and $1200 \mathrm{~s}$ of applied shear for a setup using a shear rate of $25 \mathrm{~s}^{-1}$ and surface to volume ratio of $2.5 \mathrm{~cm}^{2} / \mathrm{cm}^{3}$. Error bars represent one standard deviation.

functions obtained for a setup using a shear rate of $25 \mathrm{~s}^{-1}$ and surface to volume ratio of $2.5 \mathrm{~cm}^{2} / \mathrm{cm}^{3}$ after $0 \mathrm{~s}$ and $1200 \mathrm{~s}$ of applied shear.

Shearing appeared to result in the autocorrelation functions decaying more slowly. To quantify this, the autocorrelation functions obtained from the DLS measurements were analyzed through a cumulant analysis ${ }^{26}$ to obtain information on the average hydrodynamic radius $\left(R_{\mathrm{h}}\right)$ of mesoscale clusters in the solutions.
Weighted Least Squares Method. In order to ensure trends of increasing average hydrodynamic radius with increasing duration of applied shear were statistically significant, a weighted least-squares linear regression method was used to take the error of each average hydrodynamic radius value into consideration. The confidence intervals for the average hydrodynamic radius values found from the nonlinear regression aspect of the cumulant analysis were not symmetric, but could be made symmetric by transforming the data through the use of a power of -1 . Since the confidence intervals were based on $95 \%$ confidence (1.96 standard deviations), the standard deviation of the transformed data $\left(\sigma_{i}\right)$ could be estimated for each measurement $i$ from ${ }^{1}$

$$
\sigma_{i}=\frac{\mathrm{CI}_{\text {upper }^{-1}-\mathrm{CI}_{\text {lower }}^{-1}}^{2 \times 1.96}}{2 \times 1}
$$

Here $\mathrm{CI}_{\text {upper }}$ is the upper average hydrodynamic radius $95 \%$ confidence interval from the cumulant analysis, and $\mathrm{CI}_{\text {lower }}$ is the lower average hydrodynamic radius $95 \%$ confidence interval from the cumulant analysis.

Therefore, to simplify the weighted linear least-squares regression procedure, the confidence intervals were made symmetric and the slope of $\left(1 / R_{\mathrm{h}}\right)$ as a function of the duration of applied shear (and its error) was found for each set of conditions (see Figure 5).

For the weighted regression, the following error term was minimized. Here $y_{i}$ is based on experimental data point $i$ (reciprocal of the average hydrodynamic radius value from the cumulant analysis), $y_{i, \text { is }}$ is the fitted value for point $i$ and $w_{i}$ is the weighting of point $i . i=1,2, \ldots, n$ where $n$ is the number of data points used in the least-squares procedure. Here $w_{i}=\left(1 / \sigma_{i}^{2}\right)$.

$$
\text { error }=\sum_{i=1}^{n} w_{i}\left(y_{i}-y_{i, \mathrm{f}}\right)^{2}
$$

The data suggest with reasonable confidence that the average hydrodynamic radius increased with the duration of applied shear because the slopes in Figure 13 remain negative within their confidence intervals. Higher shear rates and surface areas resulted in a larger negative slope. This shows that growth of

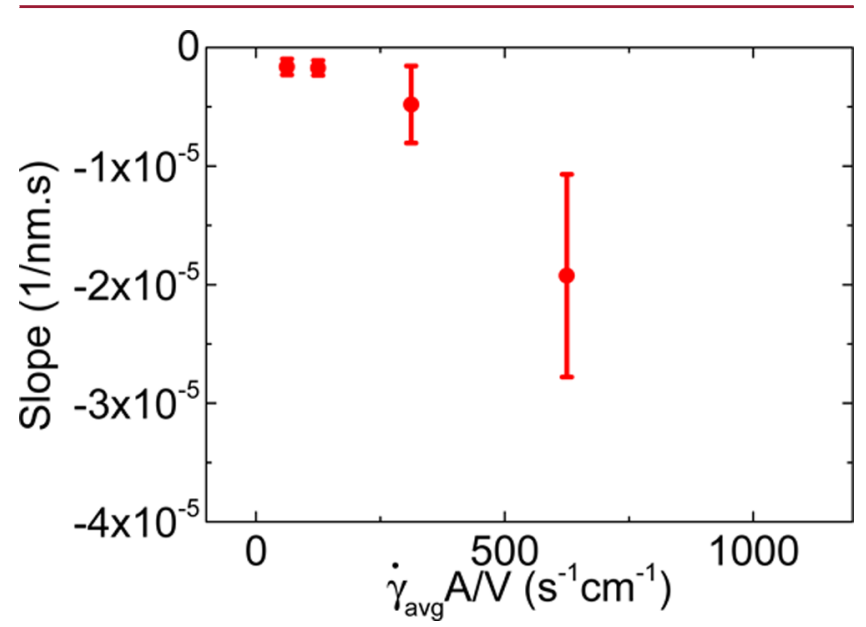

Figure 13. Slopes found from the weighted least-squares analysis of the reciprocal of the average hydrodynamic radius values plotted as a function of shear duration for each set of conditions (the conditions are represented by the product of the average shear rate and surface to volume ratio). The error bars represent $95 \%$ confidence. 
these mesoscale clusters is occurring during the induction processes and is promoted by shear and surface area. Figure 14

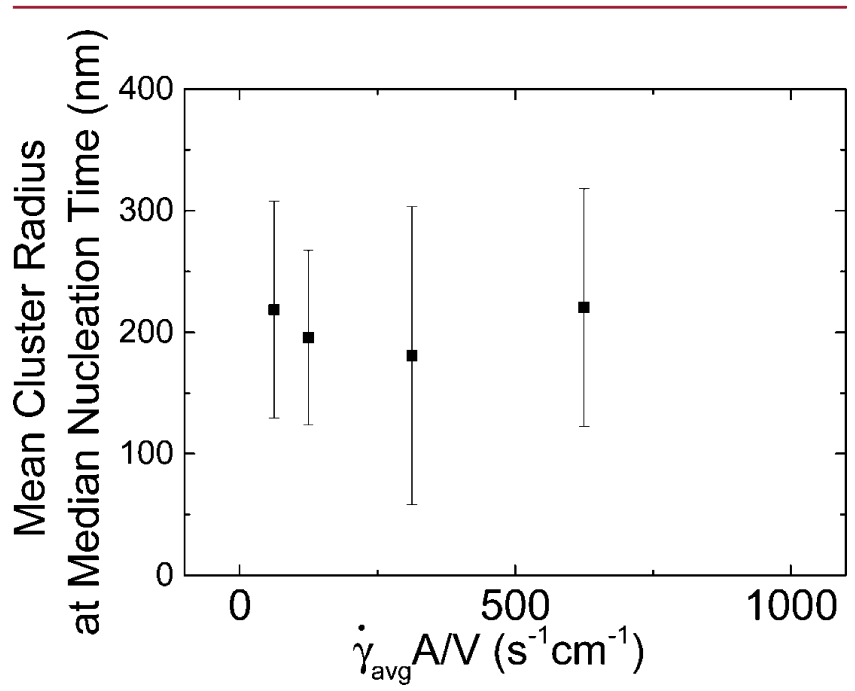

Figure 14. Average cluster radius that would be expected to occur at the median nucleation time for each set of conditions (the conditions are represented by the product of the average shear rate and surface to volume ratio). The error bars are $95 \%$ confidence intervals.

shows the average cluster size that would be expected to occur at the median nucleation time for each set of conditions. The sizes are notably larger than the size of mesoscale clusters observed under quiescent conditions, although smaller than the $750 \mathrm{~nm}$ found in a previous study. ${ }^{9}$ A Monte Carlo analysis was also carried out to further validate the trends seen, and this is detailed in the Supporting Information.

For unsheared solutions, DLS measurements showed a decay in the autocorrelation function at delay times that indicated the presence of clusters with an average hydrodynamic radius in the region of 100-150 $\mathrm{nm}$. This was similar to sizes reported in a study by Jawor-Baczynska et al., ${ }^{9}$ which found glycine solutions to contain glycine-rich clusters in the region of $125 \mathrm{~nm}$ radius. The increase in average hydrodynamic radius seen with the application of shear may have been due to the coalescence of glycine clusters, which is discussed in the following section.

Consideration of Results. The results obtained from induction time and light scattering measurements suggested that the shear rate and surface area that solutions were exposed to had an impact on the rate of nucleation. Here possible reasons for these effects are discussed.

Nucleation Rates. Shear Rate. Reasons behind the role of fluid shear in primary nucleation are not clearly understood. The increase of nucleation rate with increasing shear rate, as shown in Figure 7, could potentially be explained by several possible factors such as improved mass transfer, ${ }^{30-32}$ molecular alignment, $^{5,17,33}$ or cluster aggregation, ${ }^{3,9,20}$ resulting in higher nucleation rates. 3,9 The first two factors, namely, improved mass transfer and molecular alignment, were previously discussed by Liu and Rasmuson, ${ }^{3}$ and it was concluded that they are unlikely to explain shear rate effects on primary nucleation under common agitation conditions. We will therefore discuss the cluster aggregation factor here in further detail.

From the discussion of DLS measurement results above and a previous study on glycine, ${ }^{9}$ it has been proposed that the coalescence/aggregation of glycine mesoscale clusters may occur under stirring conditions, and larger clusters may facilitate a more rapid nucleation pathway. In order for two clusters to coalesce, they must first collide. Fluid dynamics can have a strong influence on the collision step. In the case of uniform, laminar shear flow, the collision frequency, $\varphi$, is proportional to the shear rate. ${ }^{34,35}$

$$
\varphi=\frac{4}{3}\left(r_{1}+r_{2}\right)^{3} \dot{\gamma}_{\text {avg }}
$$

Here $r_{1}$ and $r_{2}$ are the radii of the droplets to be coalesced.

This direct proportionality between collision frequency and shear rate is interesting as the induction time data indicated that the average induction times were inversely proportional to the shear rate applied. The induction times, and hence rates of primary nucleation and the growth phase, may therefore have been a function of the collision frequency of mesoscale clusters.

For an effective collision to occur, it would be expected that shear flow transport would be of at least a similar magnitude to the Brownian transport, i.e., $P e \geq 1$. This would mean that clusters can be brought together by shear at time scales shorter than it takes for them to move away from each other due to random Brownian motion. The Péclet number can be calculated from ${ }^{36}$

$$
P e^{\prime}=\frac{\dot{\gamma}_{\text {avg }} R_{\mathrm{h}}{ }^{2}}{D}
$$

Here $R_{\mathrm{h}}$ is the average radius of particles and $D$ is the corresponding diffusion coefficient.

For the conditions studied here, a Péclet number of 1 would occur for average hydrodynamic radii of $180 \mathrm{~nm}$ for a shear rate of $25 \mathrm{~s}^{-1}$ and $80 \mathrm{~nm}$ for a shear rate of $250 \mathrm{~s}^{-1}$. These length scales were of a similar magnitude to those encountered through DLS measurements (generally in the region of 100$200 \mathrm{~nm}$ radius), meaning that shear induced aggregation/ coalescence of mesoscale clusters remained a plausible explanation. Shear flow would not be expected to have a significant influence on small molecules or molecular clusters of a few nanometers.

Since the presence of larger clusters was associated with the onset of nucleation via a more rapid nucleation pathway, the larger clusters may have resulted in higher primary nucleation rates, and so the assumption of a constant nucleation rate value, $J$, for Jiang and ter Horst's model ${ }^{27}$ may not have been a valid assumption. The nucleation rate may have actually been a function of cluster size and thus a function of time, and so the log-normal distribution may be better suited for further analysis in this system. Nevertheless, since there is no detailed theory to describe the dependence of nucleation rate on cluster size, we use the Jiang and ter Horst model as a first approximation, and note that it seems to fit the observed data well.

Since the DLS results suggested that higher shear rates and surface areas resulted in a more rapid rate of increase of average hydrodynamic radius, coalescence may have been enhanced by both shear and surface. If surface-enhanced coalescence was occurring, this may account for the patterns seen in the decreasing growth time and increased nucleation rates with increased surface area. With larger surface areas, coalescence would have occurred more readily, and a sufficiently large cluster would have formed more quickly.

Surface Area. The increase of nucleation rates with increased surface areas, as shown in Figure 7, could potentially be explained in several ways. Having a larger surface area means 
there are more possible sites for heterogeneous nucleation at the glass-liquid interface, which makes the probability of a nucleation event taking place after a particular time higher when nucleation occurs heterogeneously as the critical cluster size and therefore free energy barrier for nucleation are lower according to classical nucleation theory. ${ }^{1}$ Therefore, higher nucleation rates would be expected. Alternatively, as discussed above, increased surface areas may also have enhanced the aggregation or coalescence of mesoscale clusters, with the larger clusters leading to more rapid primary nucleation. In either case, inspection of the Couette walls after an experiment suggested that some crystals grew on both the inner and outer glass walls, supporting the idea that primary nucleation may have occurred preferentially at surfaces.

Growth Times. Increased shear rates and surface areas resulted in shorter growth times, as shown in Figure 8.

Shear Rate. Higher shear rates lead to more rapid transport of the mesoscale clusters and thus more rapid aggregation or coalescence of clusters, resulting in access to faster primary nucleation pathways, as discussed above. Once primary nucleation has occurred, higher shear rates are expected to lead to faster secondary nucleation, and therefore shorter growth times are observed.

Surface Area. The observed decrease of growth time with increased surface area requires careful consideration. If multiple crystals formed during the induction time, it would be expected that the growth time would be shorter since these crystals would each eventually undergo secondary nucleation. This would lead to a more rapid increase in turbidity. With larger surface areas, the chances of multiple crystals forming would have been higher since nucleation occurs preferentially at surfaces, and indeed we observe multiple crystals formed at the glass walls at the end of our experiments.

To see whether it was likely that multiple crystals could form through primary nucleation during the induction time, first the nucleation rates and growth times obtained using Jiang and ter Horst's model were analyzed to see whether they supported the idea that multiple crystals may have formed. The product of $J V t_{\mathrm{g}}$ varied between 0.1 and 1.1 , which was of a similar order of magnitude to $J V t_{\mathrm{n}}\left(t_{\mathrm{n}}\right.$ is the nucleation time, which is equal to the measured induction time minus the time taken for crystals to reach a detectable level) values calculated (0.03-5.6). This suggested that additional crystals may have potentially formed through primary nucleation during the growth time.

\section{CONCLUSIONS}

The nucleation of glycine was investigated in supersaturated aqueous solutions exposed to well-controlled fluid shear under isothermal conditions. A wide range of average shear rates (25$\left.250 \mathrm{~s}^{-1}\right)$ and surface areas $\left(2.5-10 \mathrm{~cm}^{2}\right.$ per $\mathrm{mL}$ solution) were used, and induction times were measured. Because of the stochastic nature of nucleation, experiments were repeated multiple times. Great care was taken to eliminate any seeding in order to avoid secondary nucleation preceding the formation of first crystals through primary nucleation.

Induction times obtained under shearing were found to be considerably lower than those in unsheared solutions. The distributions of induction times were found to scale with shear rate and surface area, and scaled equations were fitted to the scaled data with low errors. In situ DLS measurements suggested that shearing caused the average size of mesoscale clusters in the solutions to increase. Larger shear rates and surface areas resulted in a faster rate of increase of average cluster size. The increases in size were thought to be due to the aggregation or coalescence of glycine mesoscale clusters.

In combination, the results suggest the following picture for primary nucleation, subsequent crystal growth, and ensuing secondary nucleation. The single dominant time scale $\left(\dot{\gamma}_{\text {avg }} A\right)^{-1}$ indicates that the process is a surface-related phenomenon, and furthermore, the dependence on shear rate suggests that shearinduced and surface-assisted aggregation or coalescence of mesoscale clusters appears to be crucial. The proposed nucleation pathway in this system includes mesoscale clusters, where primary nucleation is more likely to happen within larger clusters, regardless of whether they are located at the glassliquid interface or in the bulk. Larger shear rates and larger surface areas thus result in higher primary nucleation rates as well as shorter growth times to reach detection.

\section{ASSOCIATED CONTENT}

\section{S Supporting Information}

The Supporting Information is available free of charge on the ACS Publications website at DOI: 10.1021/acs.cgd.5b01042.

Further details of the Couette flow geometries used, the induction time measurement setup, the experimental procedures for induction time and dynamic light scattering measurements, the distributions of induction times, and a Monte Carlo method used to check the statistical trends of dynamic light scattering results (PDF)

\section{AUTHOR INFORMATION}

\section{Corresponding Author}

*Tel.: +44 (0) 141548 2410. E-mail: jan.sefcik@strath.ac.uk.

Notes

The authors declare no competing financial interest.

\section{ACKNOWLEDGMENTS}

We would like to thank James Murphy for his invaluable technical assistance and advice. Thanks to Statistics and Mathematics Advice, Research and Training (SMART) at the University of Strathclyde. The Carnegie Trust for the Universities of Scotland is acknowledged for funding the Ph.D. project of C.F. We thank the Engineering and Physical Sciences Research Council (EPSRC) for support through Centre for Innovative Manufacturing for Continuous Crystallization and Manufacturing (CMAC), Grant EP/I033459/1.

\section{NOMENCLATURE}

$A=$ total surface area of glass in contact with the solution

$c=$ concentration of glycine

$c^{*}=$ solubility of glycine

$\mathrm{CI}_{\text {upper }}=$ upper average hydrodynamic radius $95 \%$ confidence interval from the cumulant analysis

$\mathrm{CI}_{\text {lower }}=$ lower average hydrodynamic radius $95 \%$ confidence interval from the cumulant analysis

$D=$ diffusion coefficient

$g_{2}(\tau)=$ normalized intensity autocorrelation function

$J=$ nucleation rate

$N=$ rotational rate

$n=$ number of data points used in the least-squares procedure

$P\left(t_{\mathrm{i}}\right)=$ cumulative distribution of nucleation times for the Jiang and ter Horst model 
$P\left(t_{\text {scaled } \mathrm{i}}\right)=$ cumulative distribution of scaled nucleation times for the Jiang and ter Horst model $r=$ radial position

$r_{1}$ and $r_{2}=$ radii of the droplets to be coalesced

$r_{\text {in }}=$ radius of inner cylinder

$r_{\text {out }}=$ radius of outer cylinder

$R^{2}=$ coefficient of determination

$R_{\mathrm{h}}=$ hydrodynamic radius of particle

$S=$ supersaturation

$t_{\mathrm{g}}=$ growth time for nuclei to become detectable

$t_{\mathrm{i}}=$ experimentally observed induction time $\mathrm{i}$

$t_{\mathrm{n}}=$ nucleation time

$t_{\text {scaled } \mathrm{i}}=$ scaled experimentally observed induction time

$V=$ solution volume

$w_{i}=$ weighting of point $i$

$y_{i}=$ experimental data point $i$ (reciprocal of the average hydrodynamic radius value from the cumulant analysis)

$y_{i, f}=$ fitted value for point $i$

$\alpha=$ fitting parameter

$\beta=$ fitting parameter

$\gamma=$ total strain

$\dot{\gamma}=$ shear rate

$\dot{\gamma}_{\text {avg }}=$ average shear rate

$\eta=$ kinematic viscosity

$\sigma_{i}=$ standard deviation of transformed data

$\varphi=$ collision frequency

$\psi=$ scaling factor

\section{REFERENCES}

(1) Mullin, J. W. Crystallization, 4th ed.; Heineman: London, 2002.

(2) Davey, R.; Garside, J. From Molecules to Crystallizers. An Introduction to Crystallization; Oxford University Press, 2000.

(3) Liu, J.; Rasmuson, A. C. Cryst. Growth Des. 2013, 13, 4385-4394.

(4) Forsyth, C.; Mulheran, P. A.; Forsyth, C.; Haw, M. D.; Burns, I.

S.; Sefcik, J. Cryst. Growth Des. 2015, 15, 94-102.

(5) Penkova, A.; Pan, W. C.; Hodjaoglu, F.; Vekilov, P. G. Ann. N. Y. Acad. Sci. 2006, 1077, 214-231.

(6) Roberts, M. M.; Heng, J. Y. Y.; Williams, D. R. Cryst. Growth Des. 2010, 10, 1074-1083.

(7) Reis, N. M.; Chirgadze, D. Y.; Blundell, T. L.; Mackley, M. R.

Acta Crystallogr., Sect. D: Biol. Crystallogr. 2009, 65, 1127-1139.

(8) Parambil, J. V.; Schaepertoens, M.; Williams, D. R.; Heng, J. Y. Y. Cryst. Growth Des. 2011, 11, 4353-4359.

(9) Jawor-Baczynska, A.; Sefcik, J.; Moore, B. D. Cryst. Growth Des. 2013, 13, 470-478.

(10) Sypek, K.; Burns, I. S.; Florence, A. J.; Sefcik, J. Cryst. Growth Des. 2012, 12, 4821-4828.

(11) Liu, J.; Svard, M.; Rasmuson, A. C. Cryst. Growth Des. 2014, 14, $5521-5531$.

(12) Myerson, A. Handbook of Industrial Crystallization, 2nd ed.; Butterworth-Heinemann: Oxford, U.K., 2001.

(13) Kashchiev, D. Nucleation: Basic Theory and Applications; Butterworth-Heinemann: Oxford, U.K., 2000.

(14) Erdemir, D.; Lee, A. Y.; Myerson, A. S. Acc. Chem. Res. 2009, 42, 621-629.

(15) Gebauer, D.; Kellermeier, M.; Gale, J. D.; Bergstrom, L.; Colfen, H. Chem. Soc. Rev. 2014, 43, 2348-2371.

(16) Vorontsova, M.; Maes, D.; Vekilov, P. Faraday Discuss. 2015, $179,27-40$.

(17) Vekilov, P. G. Nanoscale 2010, 2, 2346-2357.

(18) Gebauer, D.; Cölfen, H. Nano Today 2011, 6, 564-584.

(19) Jawor-Baczynska, A.; Moore, B. D.; Lee, H. S.; McCormick, A.

V.; Sefcik, J. Faraday Discuss. 2013, 167, 425-440.

(20) Gebauer, D.; Volkel, A.; Colfen, H. Science 2008, 322, 18191822.
(21) Jawor-Baczynska, A.; Moore, B. D.; Sefcik, J. Faraday Discuss. 2015, 179, 141-154.

(22) Towler, C. S.; Davey, R. J.; Lancaster, R. W.; Price, C. J. J. Am. Chem. Soc. 2004, 126, 13347-13353.

(23) Taylor, G. I. Philos. Trans. R. Soc., A 1923, 223, 289-343.

(24) Devine, W.; Lowe, B. M. J. Chem. Soc. A 1971, 2113-2114.

(25) Andereck, C. D.; Liu, S. S.; Swinney, H. L. J. Fluid Mech. 1986, 164, 155-183.

(26) Pusey, P. In Neutron, X-rays and Light. Scattering Methods Applied to Soft Condensed Matter; Lindner, T. Z. P, Eds.; Elsevier: Amsterdam, 2002, Chapter 9.

(27) Jiang, S. F.; ter Horst, J. H. Cryst. Growth Des. 2011, 11, 256261.

(28) Montgomery, D.; Runger, G. Applied Statistics and Probability for Engineers, 4th ed.; John Wiley \& Sons: New York, 2006.

(29) Limpert, E.; Stahel, W. A.; Abbt, M. BioScience 2001, 51, 341352.

(30) Mullin, J. W.; Raven, K. D. Nature 1961, 190, 251-252.

(31) Mullin, J. W.; Raven, K. D. Nature 1962, 195, 35-36.

(32) Nyvlt, J.; Skrivanek, J.; Gottfried, J.; Krickova, J. Collect. Czech. Chem. Commun. 1966, 31, 2127-2136.

(33) Keller, A. Rep. Prog. Phys. 1968, 31, 623-625.

(34) Liao, Y.; Lucas, D. Chem. Eng. Sci. 2010, 65, 2851-2864.

(35) Friedlander, S. K. Smoke, Dust and Haze: Fundamentals of Aerosol Behavior; Wiley: New York, 1977.

(36) Wagner, N. J.; Brady, J. F. Phys. Today 2009, 62, 27-32. 